\title{
Influencia de la cultura organizacional en el sistema de gestión de calidad: Estado del arte.
}

\author{
Influence of the organizational culture in the quality management system: \\ State of the art.
}

Francisco Rodrigo Sanabria Estrada MSc. ${ }^{1}$, Mary Vergara Apolinario MSc. ${ }^{2}$, Mario Santacruz Mora MSc. ${ }^{3}$ \& Jorge Manuel Lucin Borbor MSc. ${ }^{4}$

\begin{abstract}
.
DOI: https://doi.org/10.33262/cienciadigital.v3i1.286

The culture of quality is a corporate tool that has been studied since the 80 s, so the objective was to determine the influence of organizational culture in the Quality Management System, according to a comparative analysis. in time, which took as reference the state of the art of other studies related to the present. Methodology of the bibliographic review was applied, whose results showed that a direct and essential link between the organizational culture that encourages and generates that the collaborators of their own will contribute with the implementation of the systems of quality management in the state of the art included between the second decade of the $21 \mathrm{st}$ century, because both evolved in the same period of time. It was concluded that both variables are associated and allow maximum satisfaction of the clients and other interested parties.
\end{abstract}

Keywords: Culture, Organizational, System, Management, Quality.

\footnotetext{
${ }^{1}$ Universidad de Guayaquil, Guayaquil, Ecuador. fsanabria74@gmail.com

${ }^{2}$ Universidad de Guayaquil, Guayaquil, Ecuador.mary.vergaraa@ug.edu.ec

${ }^{3}$ Universidad de Guayaquil, Guayaquil, Ecuador. mario.santacruz@ug.edu.ec.

${ }^{4}$ Universidad de Guayaquil, Guayaquil, Ecuador. jorge.lucinb@ug.edu.ec
} 


\section{Resumen.}

La cultura de la calidad es una herramienta corporativa que se ha estudiado a partir de la década de los 80 , por ello se planteó el objetivo de determinar la influencia de la cultura organizacional en el Sistema de Gestión de Calidad, de acuerdo a un análisis comparativo en el tiempo, que tomó como referencia el estado del arte de otros estudios relacionados con el presente. Se aplicó metodología de la revisión bibliográfica, cuyos resultados evidenciaron que un vínculo directa y esencial entre la cultura organizacional que incentiva y genera que los colaboradores por su propia voluntad contribuyan con la implementación de los sistemas de gestión de la calidad en el estado del arte comprendido entre la segunda década del siglo XXI, porque ambas evolucionaron en el mismo periodo de tiempo. Se concluyó entonces, que ambas variables están asociadas y permiten alcanzar la máxima satisfacción de los clientes y de las demás partes interesadas.

Palabras claves: Cultura, Organizacional, Sistema, Gestión, Calidad.

\section{Introducción.}

Los aportes a los sistemas de gestión de la calidad por parte de destacados autores como Eward Deming, Kaoru Ishikawa, Taichii Ono, Jenichi Taguchi, entre otros exponentes de gran relevancia en este contexto científico, evidenciaron que existen algunos elementos objetivos y subjetivos que debían coexistir para que un producto o servicio pueda satisfacer los requisitos de los clientes y de las demás partes interesadas, entre los primeros se citan los procesos y la documentación, mientras que entre los segundos se hace referencia al clima, el comportamiento y la cultura organizacional.

Precsiamente, Salcedo y Romero destacaron en su investigación que la cultura organizacional tiene implicaciones claves en la consecución de los objetivos organizacionales y de la excelencia, por esta razón, manifiestaron la relevancia del establecimiento de la misión, visión, políticas y filosofía corporativa, que conduzcan a la trasmisión de conocimientos, creencias y valores a los colaboradores, desde el mismo instante en que se incorporan como miembros de una empresa pública o privada, para que formen una cultura de calidad, cuyo impacto sea medible en la satisfacción del clinte y de las demás partes intersadas. (Salcedo \& Romero, 2014).

Las empresas que no le den importancia al desarrollo de una cultura organizacional orientada hacia la calidad, pueden ser poco competitivas, lo que estaría en inconformidad con los principios de la evoluón humana, que siempre ha mantenido la expectativa y el interés de mejorar continuamente a través del tiempo, para satisfacer requisitos individuales y colectivos y solucionar los problemas que han agobiado a la población mundial durante toda su historia. (Vesga J. , 2013). 
Por lo tanto, el desarrollo de una cultura de calidad debe ser uno de los objetivos organizacionales, debido a que si los colaboradores receptan el mensaje que la calidad es algo cotidiano y que se debe suministrar a todos los semejantes como si tratara de uno mismo, no solo que se promovería mayor competitividad en el mundo entero, sino que también se alcanzarían altos estándares de bienestar y buen vivir en el mundo entero, generándose desarrollo social y económico, también en las sociedades en vías de desarrollo.

Basado en estas aseveraciones emitidas en los párrafos anteriores de este apartado introductorio, se formuló como objetivo general del artículo, determinar la influencia de la cultura organizacional en el Sistema de Gestión de Calidad, de acuerdo con un análisis comparativo en el tiempo que se tomó como referencia del estado del arte correspondiente a otros estudios relacionados con el presente, paras el efecto, se utilizó como metodología la revisión bibliográfica, como se manifiesta en el siguiente apartado.

\section{Desarrollo.}

\section{Metodología}

La metodología aplicada en el presente estudio fue la revisión bibliográfica que facilitó la recopilación de la información que existe sobre un tema en particular, mediante la indagación de revistas, artículos cientificos, libros, diferente material archivado y trabajos académicos, mediante esta investigación proporciona una visión sobre el problema en particular.

La revisión bibliográfica del estado del arte contribuyó a determinar la conexión existente entre las variables inherentes a la cultura organizacional y los sistemas de gestión de la calidad, para identificar su relevancia en la implantación de normativas técnicas estandarizadas en las organizaciones empresariales, como es el caso, por ejemplo, de la norma ISO 9001.

Para la revisión bibliográfica, previo a la obtención de estudio inherentes al estado del arte, se seleccionó información de los textos del área en referencia, para proceder a argumentar sobre el tópico particular en análisis, enfocándose de manera particular en la cultura organizacional y en el Sistema de Gestión de Calidad, considerándose el origen, su evolución histórica y los métodos o herramientas utilizadas, efectuando la comparación de diferentes estudios sobre el tema.

\section{Definición y evolución de la cultura organizacional}

La cultura es un término muy amplio que evoca aspectos internos y externos, sociológicos y psicológicos, que evolucionó a lo largo de la historia, sin embargo, recién después de la Segunda Guerra Mundial fue objeto de estudio filosófico y científico y fue introducida en el 
sector empresarial en conjunto con la calidad, que también fue abordada como ciencia a partir de la segunda mitad del siglo XX.

El primero en abordar la cultura desde un punto de vista filosófico fue el estadounidense Thomas Kuhn, en el año 1962, quien manifestó acerca de este término lo siguiente: "representa la capacidad del ser humano para la protección y aseguramiento de su propio desarrollo, porque la cultura encierra el conocimiento, creencias, costumbres y el comportamiento adquirido por los individuos, en la sociedad en donde se desenvuelve".

Figura 1: Modelo conceptual de cultura de Charles Handy. Tomado del artículo de (Minsal \& Pérez, 2014).

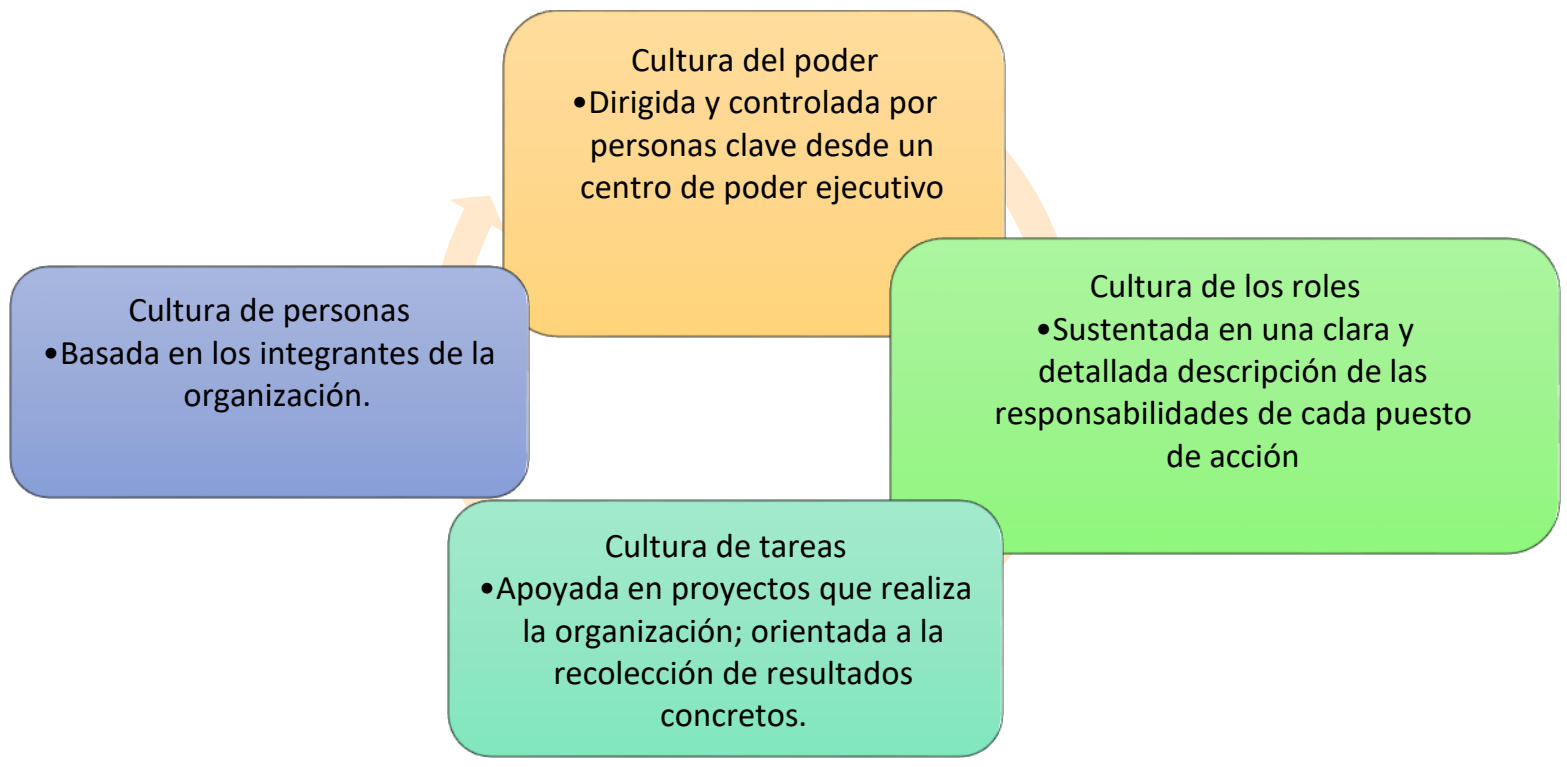

Quince años más tarde, en el año de 1977, Salzmann definió a la cultura desde una perspectiva antropológica, "como el comportamiento social adquirido por el individuo, que ha diferenciado cada generación, es decir, el modo tradicional de realizar una actividad en un espacio delimitado de la sociedad”. (Cantú, 2013).

Charles Handy en el año 1978 planteó una clasificación de la cultura organizacional, basado en cuatro elementos: poder, roles, tareas y personas; según el criterio de este experto, la cultura está controlada por un poder que lo ejerce el personal directivo, del cual depende el plan y las funciones para los demás colaboradores de la organización. Pettigrew en 1979, describió la cultura como el sistema de significados aceptados por la sociedad en un tiempo determinado. (Minsal \& Pérez, 2014).

El concepto de cultura organizacional de Charles Handy se fundamentó en primer lugar en el quehacer cotidiano de la alta dirección, representada por quienes debían conducir el 
camino hacia la excelencia, estableciendo políticas y objetivos claros que promuevan tambien una guía para que todos los demás miembros de la organización se orienten por estas políticas directrices, de modo que los procesos se realicen de manera coordinada y todos se esmeren por conseguir la máxima satisfacción de los clientes y de las partes interesadas.

En el año de 1980, Alvin Toffler dividió la historia de la humanidad en tres clasificaciones, a las que llamó olas: la sociedad agrícola-ganadera, aquella que se originó con la revolución industrial y la sociedad del conocimiento e informática, por lo que concluye que la cultura tiene carácter polisémico y heterogeneidad de acepciones. (Roselló, 2013).

Posterior a la teoría de los cuatro elementos de Charles Handy y de la concepción dada por Alvin Toffler, la evolución del término cultura se evoca en la siguiente tabla:

Tabla 1. Historia de la Cultura Organizacional.

\begin{tabular}{|c|c|c|}
\hline Año & Enfoque & Historia \\
\hline 1980 & $\begin{array}{l}\text { Simbolismo } \\
\text { Organizacional }\end{array}$ & $\begin{array}{l}\text { Dandridge, Mitroff y Joyce, incorporaron el simbolismo } \\
\text { organizacional, para concebir la cultura de los colaboradores. }\end{array}$ \\
\hline 1981 & $\begin{array}{l}\text { Primeros } \\
\text { estudios en } \\
\text { Cultura } \\
\text { Organizacional }\end{array}$ & $\begin{array}{l}\text { William Ouchi consideró que la tradición y el clima forman parte } \\
\text { intrínseca del concepto de cultura organizacional, comparando el } \\
\text { sistema empresarial norteamericano con el japonés. }\end{array}$ \\
\hline 1982 & $\begin{array}{lr}\text { Concepto } & \text { de } \\
\text { Schwartz } & y \\
\text { Davis } & \end{array}$ & $\begin{array}{l}\text { Schwartz y Davis concibieron la cultura organizacional como las } \\
\text { creencias y expectativas que comparten los colaboradores y } \\
\text { generan normas internas que conducen el comportamiento } \\
\text { individual y grupal en la empresa. }\end{array}$ \\
\hline 1982 & $\begin{array}{l}\text { Los valores y la } \\
\text { cultura }\end{array}$ & $\begin{array}{l}\text { Deal y Kennedy en } 1982 \text { enfatizaron que los valores constituyen } \\
\text { el núcleo esencial de la cultura }\end{array}$ \\
\hline 1983 & $\begin{array}{l}\text { El concepto de } \\
\text { Schein: } \quad \text { Tres } \\
\text { ángulos } \\
\text { observación de } \\
\text { la cultura }\end{array}$ & $\begin{array}{l}\text { Schein manifiesta que la cultura se asienta en tres ángulos } \\
\text { vinculados entre sí: valores, normas y creencias aceptadas y } \\
\text { practicadas por los seres humanos, en consecuencia,a través de } \\
\text { estos ángulos se pueden resolver los problemas en las } \\
\text { organizaciones }\end{array}$ \\
\hline 1990 & $\begin{array}{l}\text { Dos enfoques de } \\
\text { la Cultura } \\
\text { organizacional }\end{array}$ & $\begin{array}{l}\text { La cultura influye en el comportamiento y en el desarrollo } \\
\text { tecnológico, estructura organizacional, liderazgo y clima. } \\
\text { El enfoque funcional estima que la organización es cultura, } \\
\text { expresada como un mecanismo de comunicación socio- } \\
\text { antropológica. }\end{array}$ \\
\hline 1991 & $\begin{array}{l}\text { Cultura } \\
\text { Organizacional } \\
\text { en Colombia }\end{array}$ & $\begin{array}{l}\text { Iniciados por Coltejer, Leonisa, Carvajal, fundamentados en el } \\
\text { enfoque funcional }\end{array}$ \\
\hline
\end{tabular}


1996 Concepto de Denison estimó que la cultura organizacional se concibe de Denison diferentes maneras, según el tipo de socialización de los grupos de colaboradores, su evolución en el tiempo y en el espacio.

2007 Naturaleza de la Zapata manifestó que la cultura es aprendida de forma

Cultura inconsciente por los colaboradores, siendo sus comportamientos

Organizacional adquiridos por la repetición de un patrón.

Fuente: (González, 2007).

Hiniesto en el año 2001 clasificó a la cultura en ocho tipos, que pueden complementarse entre sí, los cuales son los siguientes:

Figura 2. Modelo de Hiniesto de clasificación de la cultura. Tomado del artículo de (Carro, Sarmiento, \& Rosano, 2017).

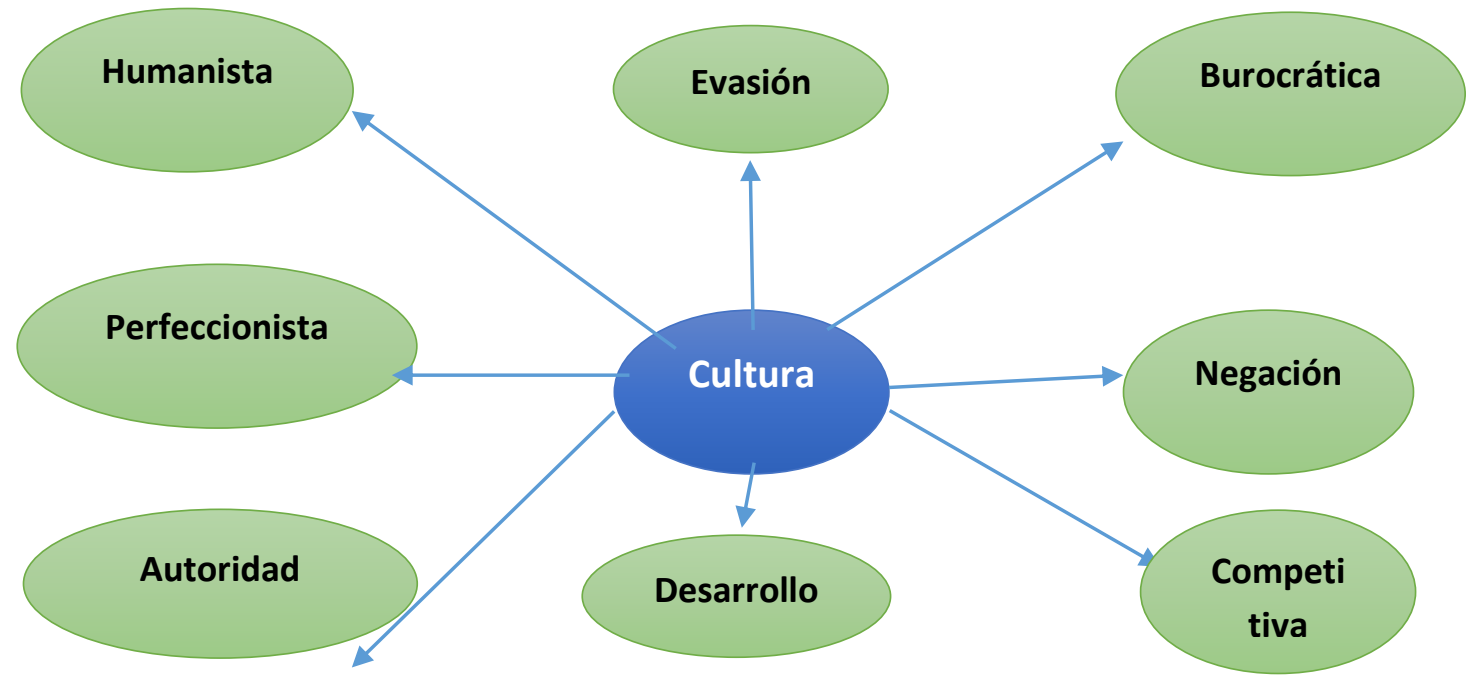

Hofstede en el año 2001, analizó la cultura fundamentándose en la actividad de cinco parámetros: distancia de poder, género, individual/colectivo, enfrentamiento de la incertidumbre, largo plazo. (Tarapuez, 2016).

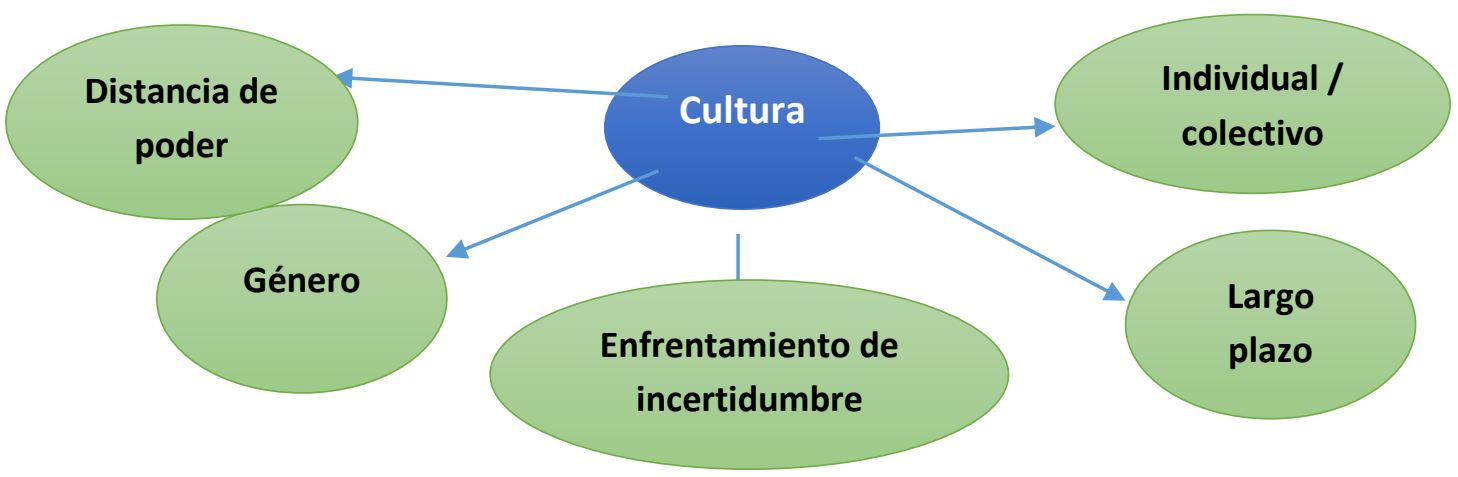


Con base en algunos crtiterios de expertos como Thomas Kuhn, Salzmann y Hofstede, Cantú (2011) definieron la cultura como "el conjunto de valores y hábitos individuales que junto con las prácticas cotidianas en el uso de las herramientas de la calidad, contribuyen a afrontar con éxito los retos organizacionales...".

La cultura es sin duda alguna, una de las dimensiones esenciales para alcanzar la excelencia en las organizaciones empresariales, es decir, que las instituciones públicas o privadas que han implementado una política que ha generado a su vez un comportamiento positivo de los colaboradores, tanto individual como grupal, fomentando un clima armónico, pueden mejorar continuamente la calidad de los procesos básicos y complementarios, cumpliendo con el principio de Edward Deming y con los postulados que se citan en las normas internacionales que rigen los sistemas de gestión de la calidad.

\section{Evolución de los sistemas de la calidad}

Kaoru Ishikawa en 1986, señaló que la calidad "inica y concluye con la preparación intelectual, sentimental y de valores de los colaboradores, para sacar el máximo provecho del esfuerzo de cada trabajador, además del mantenimiento de controles eficientes en las diferentes etapas de los procesos", mientras que Edward Deming en 1989 concibió la calidad "como la traducción de las características medibles de las necesidades que presentan los usuarios o clientes, cuyos hallazgos deben servir de guía para la toma de decisiones hacia la mejora continua". Para Taichii Ono, la calidad es "la aplicación del sentido común". (Lozano, 1998).

A lo largo de la historía, los expertos que investigaron sobre la calidad, proporcionaron diferentes aportes sobre esta área organizacional, en donde cada uno emitió criterios de acuerdo a la investigación desarrollada, evolucionando la calidad desde la inpección en el siglo XIX, hasta llegar a los sistemas de gestión de la calidad actual, para lo cual se diseñó el siguiente gráfico:

Figura 3. Etapas de la evolución de la calidad. Nota: Tomado del artículo de (Torres, Ruiz, Solís, \& Martínez, 2012).

Primera etapa Control de la calidad por inspeccion. Siglo XIX

Segunda etapa. Control estadístico de procesos. 1930 a 1950.

Tercera etapa. Aseguramiento de la calidad, calidad total. 1950 a 1970.

Cuarta etapa. Administración total de la calidad. 1980.

Quinta etapa. Gestión de la calidad. 1987. Norma ISO 9001. 
La calidad, a lo largo de la historia se manifestó con diferentes expresiones y metodologías, siendo la primera etapa la inspección creada en el siglo XIX, donde la producción y comercialización se realizaban en las empresas que surgieron a partir de la revolución industrial; mediante este método se buscaba medir o identificar ciertos defectos del producto final. (Montaudon, 2015).

En la segunda etapa tuvo apogeo el control estadístico de la calidad, desde 1930 hasta 1950, donde el eje central de este método se centró en la identificación y eliminación de las causas de los defectos del producto, mediante el muestreo de lotes, cuya herramienta fue adoptada por los países que participaron en la Segunda Guerra Mundial. (Torres, Ruiz, Solís, \& Martínez, 2014).

La tercera etapa se denominó como aseguramiento de la calidad, desde 1950 hasta 1970, cuya característica principal era que el producto debía cumplir con los requisitos para su introducción en el mercado, en esta etapa surge el mejoramiento continuo de Edward Deming y también se creó el sistema de calidad total en Japón. (Montaudon, 2015).

La cuarta hizo referencia a la administración de la calidad total, en 1980, pasando de ser una prioridad competitiva para convertirse en un requisito indispensable. posteriormente se introdujo la normativa ISO, dando origen a la quinta etapa de la calidad, hecho acaecido en el año 1987, en la cual ha predominado el concepto de estandarización de los procesos siendo la primera norma creada, aquella de la familia ISO 9001. (Torres, Ruiz, Solís, \& Martínez, 2014).

\section{Sistema de Gestión de la Calidad}

Un Sistema de Gestión de la Calidad se concibe como el método mediante el cual la organización planifica, ejecuta, controla y define la actividad preventiva, para cumplir con las metas y objetivos establecidos en el plan estratégico y en la misión organizacional, facilitando el suministro de productos o servicios de excelente calidad que satisfagan los más exigentes requisitos de los clientes y de las partes interesadas. (Vizurraga, 2014).

El Sistema de Gestión de la Calidad surgió en el año 1987 con la norma ISO 9001, la misma que desde sus inicios fue aplicada por las diferentes organizaciones en todo el mundo para estandarizar sus procesos, con el fin de ofrecer productos y servicios de excelente calidad de manera estable y permanente, a la vez de optimizar sus actividades e incrementar su eficiencia, siendo la primera norma ISO creada en el año 1987, cuya característica fue la conformidad, debido a que su antecedente fue la norma MIL-Q-9858, cuyo fundamento fue la conformidad y no la mejor continua. (Del Castillo \& Sardi, 2014).

Esta situación generó cambios en la norma ISO 9001, siguiendo su revisión en el año 1994, cuando la normalización se centró en la mejora continua y en la satisfacción del cliente, antes 
que solamente en la conformidad. En el año 2000 se realizó la segunda revisión de la norma de gestión de calidad, introduciéndose el enfoque de procesos, siendo el propósito de la gestión alcanzar los procesos previstos y documentar los procedimientos en la medida necesaria para efectuar la gestión. En el 2008 se llevó a cabo la tercera revisión, dentro de esta versión se colocó al cliente como centro de importancia y relevancia, estableciendo que el proveedor debe definir de forma clara el rol a cumplir, para identificar a los clientes, usuarios o potenciales clientes, definiendo sus necesidades reales, mediante está certificación se buscaba garantizar la calidad de productos y servicios mejorando los procesos y la imagen corporativa. (Roncancio, Castro, \& Rivera, 2015).

Figura 4 Sistema de Gestión de la calidad. Nota: Tomado del artículo de (Arraut, 2010).

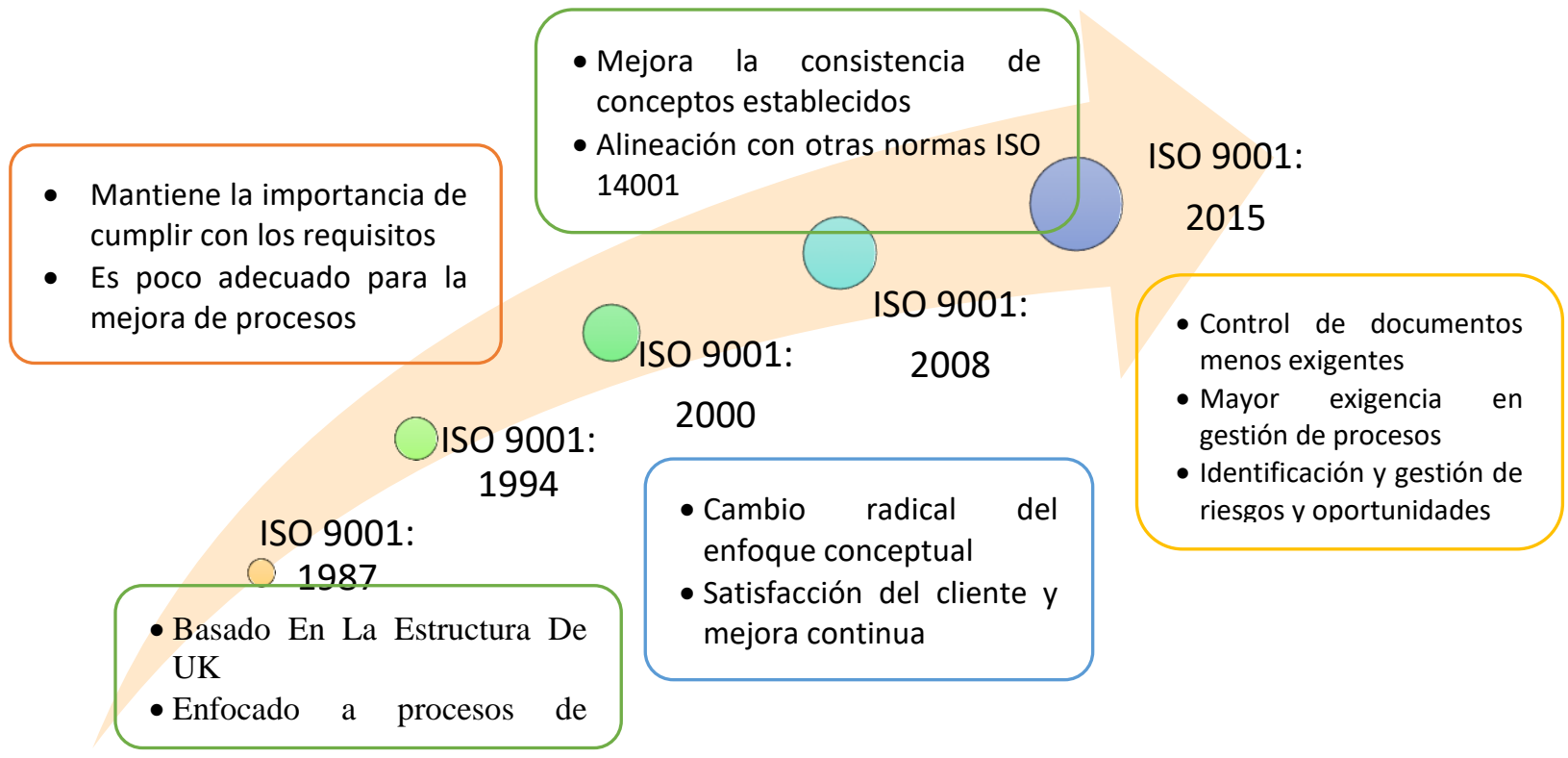

Siguiendo con la evolución del Sistema de Gestión de calidad en el 2015 se efectuó la última revisión, en esta actualización se propuso mayor libertad a la adaptación del sistema en análisis dentro de las empresas y procesos, a través de esta versión no se considera el sistema de gestión de calidad como un propósito específico sino la herramienta para la innovación, evidenciándose algunas mejoras como el interés con el que se trata a los usuarios, consumidores y organismos reguladores, contemplando como objetivo cubrir las necesidades de los clientes mediante el ciclo de planificar - hacer - verificar y actuar reconociendo los riesgos y el impacto en la capacidad de la organización en la entrega de productos y servicios. (Arraut, 2010).

Dentro de esta versión no se especifica un procedimiento de documentación, reflejando mayor flexibilidad en este aspecto, un punto de transición se presentó en la ISO 9001: a partir de septiembre 2015 y hasta septiembre del 2018 destinada a la fase de las organizaciones 
certificadas; en un período de tres años, dónde podrán coexistir dos versiones hasta que se presente una nueva actualización.

\section{La influencia de la cultura organizacional en los sistemas de gestión de la calidad, de conformidad al estado del arte}

Definida la cultura organizacional y los sistemas de gestión de la calidad, el artículo prosigue con la determinación cómo influye la primera a la segunda variable en mención, esto significa, que para cumplir con el objetivo central del estudio, ha sido necesario tomar como referencia varios estudios relacionados, para corroborar que la cultura es un componente esencial de los sistemas de gestión de la calidad, sin el cual no se podría lograr la excelencia, a pesar de que la corporación cuente con la preparación del talento humano, el desarrollo tecnológico y la documentación correspondiente.

A partir de esta idea que se plantea defender como parte del desarrollo del artículo, se ha analizado los siguientes estudios relacionados que forman parte del estado del arte de la segunda década del siglo XXI, los cuales se presentan en los siguientes párrafos de este apartado.

(Vivanco, Enríquez, \& González, 2013), elaboró el estudio titulado "los sistemas de control de gestión y la cultura organizacional en las PYMES" el cual estuvo enfocado en identificar una alternativa de mejora del desempeño de las PYMES para propiciar su subsistencia, donde se tomó el modelo de Cameron y Quinn (1999) para identificar el tipo de cultura adoptada en estas empresas, cuyos resultados arrojaron alta significancia entre las variables inherentes a la cultura organizacional y el desarrollo de la calidad, porque la primera en mención es un factor clave para adoptar los sistemas de control de gestión en las organizaciones.

(Guillén \& Aduna, 2014) en su trabajo plantearon como objetivo demostrar la influencia de la cultura sobre el clima organizacional en las empresas, mediante el análisis organizacional y el estudio de caso comparativo de medianas empresas, cuyos resultados evidenciaron que algunas entidades proporcionaron elementos de análisis para una mejor comprensión del clima organizacional, ayudando a esclarecer de este modo las causas de la satisfacción laboral, reconociendo que no todas las organizaciones tienen el mismo comportamiento organizacional, sugiriéndose la búsqueda y atención de las particularidades que hacen la diferencia, para que se implemente y perdure la implementación de sistema de calidad.

El trabajo realizado por (Salcedo \& Romero, 2014) denominado "cultura organizacional y gestión de la calidad en una empresa del estado venezolano", el cual tuvo como objeto identificar los factores de la cultura organizacional que facilitan la implantación del sistema de gestión de la calidad en la corporación en mención, para el efecto, fueron analizadas las variables de la filosofía de gestión, el estilo gerencial y la cultura organizacional, obteniéndose como resultado que es necesario que las instituciones investiguen y fortalezcan 
las cualidades humanas que deben internalizar los principales actores hacia la calidad, de acuerdo a estándares internacionales, siendo necesario el trabajo en equipo, la reducción de los niveles jerárquicos en la compañía y el mejoramiento de la comunicación interna, cuya acción gerencial debe orientarse hacia la gestión de la calidad.

(Vesga J. , 2015) realizó un estudio denominado "cultura organizacional y sistemas de gestión de la calidad: una relación clave en la gestión de las instituciones de educación superior", el cual presenta una discusión sobre la importancia de la cultura organizacional en la implementación de sistemas de gestión de la calidad como un componente clave para elaborar los planes previo a su implementación, con el objetivo de alinear la cultura mediante estrategias de gestión e integrarla, con la expectativa de cubrir las demandas y expectativas de las sociedades actuales, como parte de su particularidad e identidad y en fiel cumplimiento de las normas ISO 9001.

(Soracipa, 2015), analizó el tema "definición de estrategias de transformación de la cultura organizacional en función de un sistema de gestión de calidad”, en el cual se presentó una propuesta metodológica para la incorporación de los principios de un sistema de gestión de calidad basado en el modelo ISO 9001, enfocado en promover el funcionamiento exitoso y la sostenibilidad, para incorporar los principios de calidad en la cultura organizacional, basado en el análisis y relación entre los conceptos de la cultura de la calidad y aspectos identificados en la norma ISO 9001.

El articulo realizado por (Marulanda, López, \& Castellanos, 2016), denominado "la cultura organizacional y su influencia en las buenas prácticas para la gestión del conocimiento en las PYMES de Colombia", el cual tuvo como objetivo la exposición de la relación existente entre la cultura organizacional y las buenas prácticas para la gestión del conocimiento mediante un modelo de evaluación de gestión del conocimiento aplicado a 321 empresas, el cual dio como resultado que la cultura organizacional influyó de forma positiva en las buenas prácticas de la gestión del conocimiento, con la propuesta de incentivos que signifiquen beneficios para los clientes internos, generando niveles de compromiso para la mejora continua.

(Barahona \& Rodríguez, 2017), en su estudio denominado "la cultura organizacional, un camino para humanizar la implementación del sistema de gestión de calidad - ISO 9001:2008, trazó como objetivo la elaboración de una propuesta metodológica para el fortalecimiento de la cultura organizacional y la implementación de Normas Técnicas de Calidad como ISO 9001:2000, en el cual fueron analizados cuatro referentes, tales como: valores organizacionales, formas de percepción, clima organizacional y la norma técnica, cuyos hallazgos evidenciaron la necesidad de introducir el entramado cultural de las organizaciones que facilite la implementación de sistemas de gestión de la calidad, razón por la que se propuso no instrumentalizar a los seres humanos sino sugerir su humanización. 
Tabla 2. Artículos que relacionan la Cultura Organizacional y el Sistema de Gestión de la Calidad, según el estado del arte.

\begin{tabular}{lll}
\hline Año & Autor & Resultados \\
\hline 2012 & Martínez \& & Es necesario el alineamiento de una cultura organizacional antes de \\
& Robles & la implantación de los sistemas de gestión para atender las \\
& & necesidades que requiere la organización.
\end{tabular}

2012 Caicedo \& Al promover activamente la realización de programas encaminados a Caldas la creación de una cultura de calidad y mejoramiento continuo, se facilite la implementación y mantenimiento de los sistemas de gestión de calidad y se fortalece el bienestar y la calidad de vida de la población.

2013 Charón Se establece la necesidad de incorporar un sistema de gestión, mediante el diseño estratégico donde se consideraron las macro variables para la planificación, en referencia a las dimensiones económicas y sociales que condicionan su comportamiento de los colaboradores

2013 Vivanco, La cultura organizacional es un factor clave para adoptar los sistemas Enríquez, de control de gestión en las organizaciones

$\&$

González

2014 Guillen \& Las empresas proporcionan elementos de análisis para una mejor Aduna comprensión del clima organizacional ayudando a esclarecer, las causas de la satisfacción laboral, reconociendo que no todas las organizaciones tienen el mismo comportamiento organizacional razón por la que se requiere buscar y atender las especificidades que hacen la diferencia, para la implementación de sistema de calidad, de modo que este perdure.

2014 Salcedo \& Es necesario el trabajo en equipo, la reducción de los niveles Romero jerárquicos en la compañía y el mejoramiento de la comunicación interna para fortalecer la cultura organizacional, y orientar adecuadamente la gestión de la calidad.

2015 Vesga La implementación de los sistemas de gestión de la calidad resulta de gran importancia para desarrollar condiciones óptimas que cubran las demandas y expectativas de las sociedades actuales, cuyas características se encuentran establecidas en las normas ISO 9001.

2015 Soracipa El funcionamiento exitoso y la sostenibilidad, para incorporar los principios de calidad en la cultura organizacional, se basan en el análisis y relación entre los conceptos de cultura organizacional, 
cultura de la calidad, como aspectos identificados en las normas ISO 9001.

2016 Marulanda, La cultura organizacional influye de forma positiva en las buenas

López, \& prácticas de la gestión del conocimiento, que sumado a la propuesta

Castellanos de incentivos pueden significar beneficios para los mismos, para la generación de altos niveles de compromiso.

2017 Barahona Existe la necesidad de introducir el entramado cultural de las \& organizaciones, para la implementación de los sistemas de gestión de Rodríguez. la calidad, razón por la cual se propuso no instrumentalizar a los seres humanos, sino que se sugiere su humanización.

Fuente: Elaboración propia.

\section{Mecanismos para el desarrollo de la cultura organizacional y su impacto en los sistemas} de gestión de la calidad

La teoría sobre la cultura organizacional, no establece mecanismos específicos para el desarrollo de una cultura organizacional, aunque algunos autores que estudiaron este componente de la administración de empresas y de la gestión de talento humano, lo asociación a la política de personal y al liderazgo. (Vesga J. , 2013).

De esta manera, algunos de los factores que tienen vínculos con el desarrollo de una cultura de calidad, son los siguientes:

Figura 5. Factores para el desarrollo de una cultura de calidad, según fuente de (Camisón \& González, 2007) y (Salcedo \& Romero, 2013).

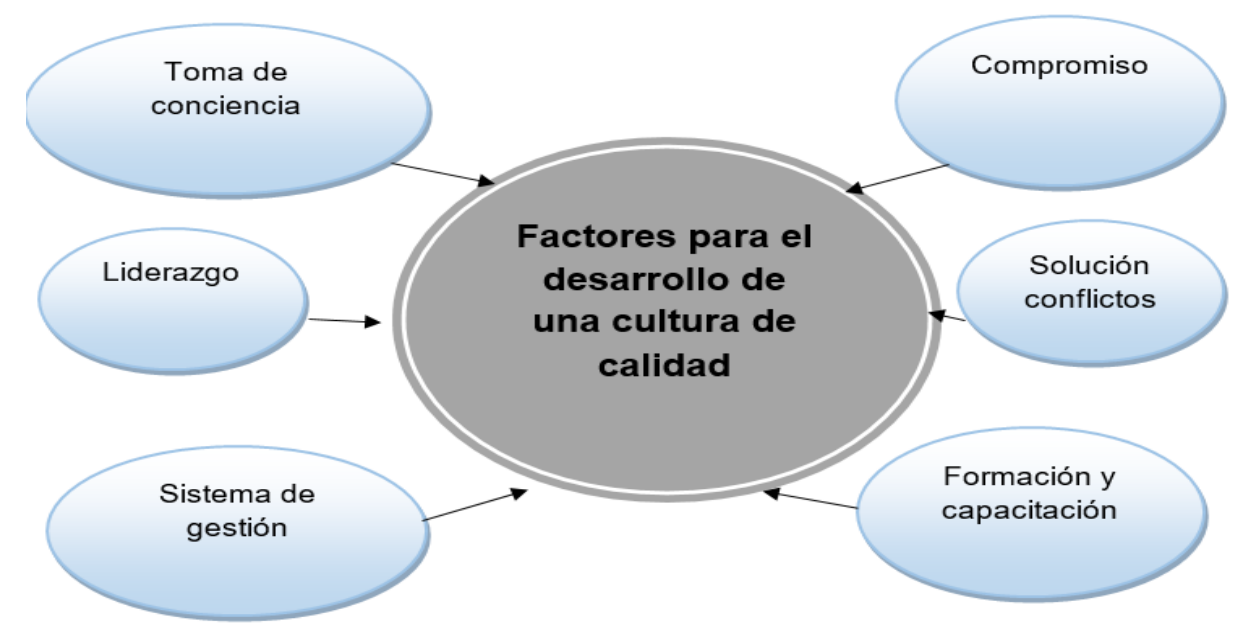


Sin embargo, el problema radica en cómo lograr que los trabajadores adquieran el compromiso para que tenga lugar el desarrollo de una cultura de calidad. Al respecto, el ciclo de Edward Deming y sus catorce principios, establecen los hábitos de calidad, entre los que se citan hacer bien las cosas a la primera vez, mantener altos niveles de proactividad, de sinergia, afilar la sierra, los cuales además conducen a la mejora continua. Algunos autores, como (Hernández A. , 2016) se refieren a la cultura organizacional mediante el Modelo de Investigación - Acción, cuya aplicación se fundamenta en la aplicación de los siguientes pasos:

- Identificación de la cultura actual

- Contratación de asesores o expertos en el tópico de la cultura organizacional.

- Recopilación de información.

- Diagnóstico.

- Retroalimentación del personal.

- Plan de acción.

- Ejecución del plan de acción.

- Evaluación de la alternativa de solución ejecutada.

- Mejora continua con acciones correctivas y preventivas. (Hernández A. , 2016).

Lewin creó en 1991 un modelo para el desarrollo de una cultura de calidad en las organizaciones, el cual se fundamenta en tres componentes: descongelamiento, movimiento y recongelamiento. En la segunda etapa (movimiento) es donde la administración principal introduce los mecanismos para que el personal adquiera el compromiso y en la última fase (recongelamiento), este mecanismo, valga la redundancia, se convierte en un hábito.

Edgar Schein también creó un método en 1993, donde agrupa los elementos en primarios y secundarios, los básicos están vinculados a la creación de la compañía y los secundarios al desarrollo de la cultura en una etapa posterior. (Naranjo, Jiménez, \& Sanz, 2012).

La propuesta de Will y Solum creada en el año 1994, establece que el desarrollo de la cultura de calidad en las empresas se fundamenta en "leyes naturales" adscritas a las organizaciones, coincidiendo con Lewin en que la cultura se fomenta paso a paso, en el largo plazo, desarrollando hábitos en la etapa de la implementación, donde se establecen los mecanismos de la formulación de la política, formación, capacitación y motivación. (Palacios, 2013).

La experiencia del autor de este artículo en la administración de una institución hospitalaria de gran reconocimiento en la localidad, permite coincidir con la teoría del largo plazo para el desarrollo de una cultura de calidad, que sí es posible, siempre y cuando el liderazgo constituya un ejemplo para todo el personal, porque los procesos de formación, capacitación y motivación, no siempre arrojan resultados en cortos periodos de tiempo, menos aún para cambiar culturas que vienen impregnadas desde el hogar en la niñez de los colaboradores. 
Por consiguiente, el liderazgo directivo con valores y buena actitud, puede transmitir las mismas virtudes y confianza al personal, para que a través del proceso educativo y de inducción continua, que incorpore los incentivos y la democracia para escuchar las aspiraciones del talento humano y solucionar la causa de los conflictos que se pueden generar en organizaciones como las hospitalarias, donde el clima por lo general suele ser tenso, se puede contribuir al desarrollo de una cultura de calidad en el largo plazo.

\section{Conclusiones.}

- La revisión de teorías correspondientes a la evolución de la cultura organizacional y los sistemas de gestión de la calidad, evidenciaron que las investigaciones sobre la primera variable se fortalecieron en la década de los 80 , el mismo periodo en que nacían los sistemas de gestión de la calidad con la incorporación de la primera norma ISO 9001 en el contexto mundial en 1987, estableciéndose una relación directa entre ambas herramientas, por lo que algunos autores como Schein, Hofstede y Cantú las unieron creando el término cultura de calidad.

- Al repasar el estado del arte relacionado con las dos variables a las que se hace referencia en el presente artículo, se pudo corroborar que la cultura organizacional es un elemento clave sin el cual no se puede implementar adecuadamente ni mantener un sistema de gestión de la calidad, aunque la entidad cuente con documentación, tecnología de punta y personal preparado.

- Se concluyó que, en efecto, las empresas deben desarrollar una cultura de calidad que facilite la implementación del sistema de gestión de la calidad, al lograr que los colaboradores contribuyan por su propia voluntad con el cumplimiento de los requisitos y sumen esfuerzos conjuntos en equipo, para alcanzar los máximos estándares de la norma técnica ISO 9001, promoviendo de esta manera la máxima satisfacción de los clientes y de las demás partes interesadas.

\section{Referencias bibliográficas.}

Arraut, L. (2010). La Gestión de calidad como innovación organizacional para la productividad de la empresa. Scielo, 20-41. Obtenido de http://www.scielo.org.co/pdf/ean/n69/n69a03.pdf

Barahona, A., \& Rodríguez, D. (2017). La cultura organizacional, un camino para humanizar la implementación del sistema de gestión de calidad - ISO 9001:2008. SIGNOS Investigación en sistemas de gestión, $154-162$. Obtenido de https://www.researchgate.net/publication/318486783_La_cultura_organizacional_u n_camino_para_humanizar_la_implementacion_del_sistema_de_gestion_de_calida d_-_ISO_90012008

Camisón, C., \& González, T. (2007). Gestióin de la calidad, conceptos, enfoques, mdelos y sistemas. Madrid: Pearson Educacion. 
Cantú, H. (2011). Desarrollo de una cultura de calidad (Cuarta ed.). México: Mc Graw Hill. Cantú, H. (2013). Calidad - hábito para la competitividad. México: Tecnológico de Monterrey.

Carro, J., Sarmiento, S., \& Rosano, G. (2017). La cultura organizacional y su influencia en la sustentabilidad empresarial. La importancia de la cultura en la sustentabilidad empresarial. Estudios Genrenciales, http://www.elsevier.es/es-revista-estudiosgerenciales-354-articulo-la-cultura-organizacional-su-influenciaS0123592317300773.

Del Castillo, A., \& Sardi, N. (2014). Las normas ISO y el concepto de calidad aplicado a los servicios médicos en anestesiología. Revista Colombiana de Anestesiología, 40(1), 16.

González, J. (2007). Historia de la Cultura Organizacional. Universidadviu.com, https://www.preceden.com/timelines/247120-historia-de-la-cultura-organizacional.

Guillén, I., \& Aduna, A. (2014). La influencia de la cultura y del estilo de gestión sobre el clima organizacional. Estudio de caso de la mediana empresa en la Delegación IZTAPALAPA. Universidad Autónoma Metropolitana, 42-58. Obtenido de https://www.icesi.edu.co/revistas/index.php/estudios_gerenciales/article/view/253/1 192

Hernández, A. (2016). Aproximación teórica a modelo de cambio planeado de gestión organizacional para la innovación educativa desde la Teoría de la Complejidad y Empowerment. Educación en Contexto, 2(1), 17.

Lozano, L. (1998). ¿Qué es calidad total? Scielo Perú, http://www.scielo.org.pe/scielo.php?pid=S1018130X1998000100006\&script=sci_arttext.

Marulanda, C., López, M., \& Castellanos, J. (2016). La cultura organizacional y su influencia en las buenas practicas para las buenas practicas para la gestión del conocimiento en las PYMES de Colombia. AD-minister, pp. 163-176. Obtenido de http://www.scielo.org.co/pdf/adter/n29/n29a08.pdf

Minsal, D., \& Pérez, Y. (2014). Hacia una nueva cultura organizacional: la cultura del conocimiento. Scielo, 16(3), 15.

Montaudon, C. (05 de 05 de 2015). Explorando la noción de calidad. Acta Universitaria, 20(2), 10.

Naranjo, J., Jiménez, D., \& Sanz, R. (2012). Es la cultura organizativa un determinante de la ionnovación en la empresa. Madrid: Cuadernos de Economía y Dirección de la Empresa.

Palacios, P. (2013). La sustentabilidad y la responsabilidad social inciden y transforman nuestra cultura organizacional y nuestra forma de trabajar. México: e-book.

Roncancio, M., Castro, J., \& Rivera, A. (2015). Análisis comparativo de las normas ISO 9001:2008, ISO. Respuestas, 20(1), 17. 
Roselló, D. (2013). Diseño y gestión de proyectos culturales. Barcelona, España: Nexe Cultural.

Salcedo, I., \& Romero, J. (2013). Cultura Organizacional y Gestión de la Calidad en una Empresa del Estado venezolano. Revista venezolana de Gerencia, 11(33), 15.

Salcedo, I., \& Romero, J. (2014). Cultura Organizacional y Gestión de la Calidad en una Empresa del Estado venezolano. Revista Venezolana de Gerencia, pp. 74-82. Obtenido de Revista Venezolana de Gerencia [online]: http://www.scielo.org.ve/scielo.php?script=sci_arttext\&pid=S131599842006000100005\&lng=es\&nrm=iso>. ISSN 1315-9984.

Soracipa, N. (2015). Definición de estrategias de transformación de la cultura organizacional en función de un sistema de gestión de calidad. SIGNOS, pp.139-152.

Tarapuez, E. (07 de 07 de 2016). Las dimensiones culturales de Geert Hofstede y la intención emprendedora en estudiantes universitarios del departamento del Quindío (Colombia). Pensamiento y Gestión, 1(41), 30.

Torres, K., Ruiz, T., Solís, L., \& Martínez, F. (2, Julio - Diciembre de 2012 de Julio Diciembre de 2012). Calidad y su evolución: una revisión. Dimensión empresarial, págs. 100-107. Obtenido de Quality and its evolution: a review.

Torres, K., Ruiz, T., Solís, L., \& Martínez, F. (06 de 06 de 2014). Calidd y su evolución: una revisión. Dimensión Empresarial, 10(2), 10.

Vesga, J. (2013). Cultura organizacional y sistemas de gestión de la calidad: una relación clave en la gestión de las instituciones de educación superior. Revista Guillermo de Ockham, 11(2), 12.

Vesga, J. (2013). Cultura organizacional y sistemas de gestión de la calidad: una relación clave en la gestión de las instituciones de educación superior. Revista Guillermo de Ockham, pp. 89-100.

Vesga, J. (2015). Cultura organizacional y sistemas de gestión de la calidad: una relación clave en la gestión de las instituciones de educación superior. Revista Científica Guillermo de Ockham, pp. 89-100.

Vivanco, J., Enríquez, L., \& González, M. (2013). Los sistemas de control de gestión y la cultura organizacional en las pymes. Mercados y Negocios, 55-72.

Vizurraga, Á. (2014). Las normas internacionales ISO y la globalización del mercado. Lima, Perú: GRAFICARTE, INIA, Cámara Forestal, proyectos PD 152/91.

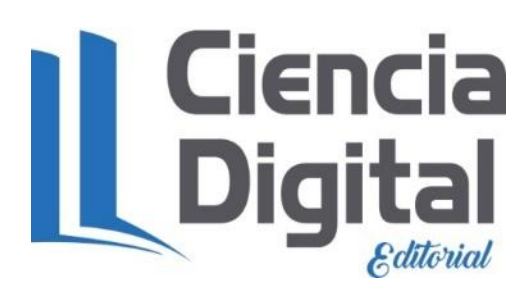


Para citar el artículo indexado.

Sanabria F., Vergara M., Santacruz M. \& Lucin J. (2019). Influencia de la cultura organizacional en el sistema de gestión de calidad: Estado del Arte. Revista electrónica Ciencia Digital 3(1), 239-256. Recuperado desde: http://cienciadigital.org/revistacienciadigital2/index.php/CienciaDigital/article/view/286/68 5

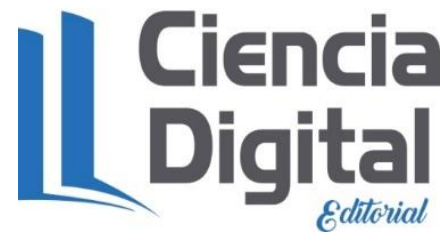

El artículo que se publica es de exclusiva responsabilidad de los autores y no necesariamente reflejan el pensamiento de la Revista Ciencia Digital.

El artículo queda en propiedad de la revista y, por tanto, su publicación parcial y/o total en otro medio tiene que ser autorizado por el director de la Revista Ciencia Digital.
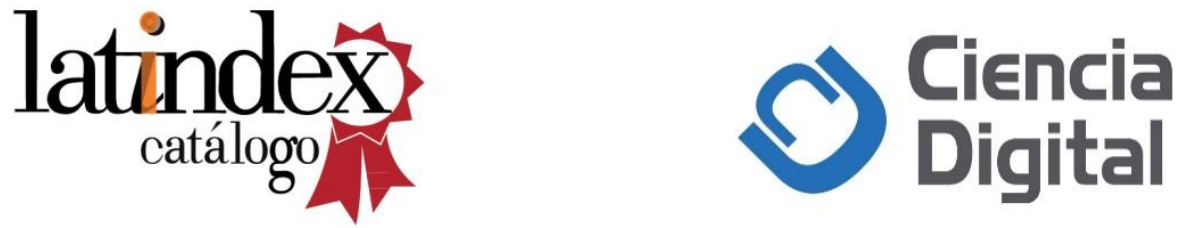